\title{
Mutual Storage Mode Ion/Ion Reactions in a Hybrid Linear Ion Trap
}

\author{
Yu Xia, Jin Wu, and Scott A. McLuckey \\ Department of Chemistry, Purdue University, West Lafayette, Indiana, USA \\ Frank A. Londry and James W. Hager \\ MDS SCIEX, Concord, Ontario, Canada
}

Ion/ion proton transfer reactions involving mutual storage of both ion polarities in a linear ion trap (LIT) that comprises part of a hybrid triple quadrupole/linear ion trap mass spectrometer have been effected. Mutual ion storage in the $x$ - and $y$-dimensions arises from the normal operation of the oscillating quadrupole field of the quadrupole array, while storage in the z-dimension is enabled by applying unbalanced radio-frequency amplitudes to opposing sets of rods of the array. Efficient trapping $(>90 \%)$ is achieved for thermalized ions over periods of several seconds. Reactions were demonstrated for multiply charged protein/peptide cations formed by electrospray with anions derived from glow discharge ionization of perfluoro(methyldecalin) (PMD) introduced from the side of the LIT rod array. Doubly and singly charged protein ions are readily formed via ion/ion reactions. The parameters that affect ion/ion reactions are discussed, including the degree of RF unbalance on the LIT rods, vacuum pressure, nature of the buffer gas, reaction time, anion abundance, and the low mass cutoff for ion/ion reaction. The present system has a demonstrated upper mass-to-charge ratio limit of at least 33,000. The system also has high flexibility with respect to defining $\mathrm{MS}^{\mathrm{n}}$ experiments involving both collision-induced dissociation (CID) and ion/ion reactions. Experiments are demonstrated involving beam-type CID in the pressurized collision quadrupole (Q2) followed by ion/ion reactions involving the product ions in the LIT. Ion parking experiments are also demonstrated using the mutual storage ion/ion reaction mode in the LIT, with a parking efficiency over 60\%. (J Am Soc Mass Spectrom 2005, 16, 71-81) (c) 2004 American Society for Mass Spectrometry

$\mathrm{T}$ The capability of the electrodynamic ion trap for storing simultaneously both positively-charged and negatively-charged ions [1] and its ability to execute multiple step experiments $[2,3]$ make it particularly well-suited for conducting sophisticated experiments involving ion/ion reactions. To date, most applications of ion/ion reactions in quadrupole ion traps have emphasized the controlled charge reduction of multiply charged positive or negative ions [4]. Charge state manipulation has served three major purposes in these studies: mixture analysis [5-9], especially with the application of the "ion parking" [10] technique for gas-phase concentration and purification [11, 12], the formation of ions that cannot be directly produced by ESI for subsequent tandem mass spectrometry studies [13-15], and the reduction of the product ion charge states to singly and doubly charged so as to simplify identification of the product ions [11,12, 16-19].

While three-dimensional quadrupole ion traps have

Published online December 13, 2004

Address reprint requests to Dr. S. A. McLuckey, Department of Chemistry, Purdue University, West Lafayette, IN 47907-2084, USA. E-mail: mcluckey@purdue.edu been demonstrated to be well-suited for conducting sophisticated experiments involving ion/ion reactions, they suffer from limited ion storage capacity and relatively low trapping efficiencies, typically $1-5 \%$, for externally generated ions [20, 21]. Linear ion traps (LITs), on the other hand, provide greater ion storage capacity than three-dimensional ion traps of comparable inscribed radius and offer significantly better trapping efficiencies for externally injected ions [20, 22]. While the LIT has been known since the quadrupole storage ring was introduced [23], it was not until recently that LITs were developed as stand-alone mass spectrometers [22], or as part of a triple quadrupole instrument [20, 24-26]. The latter "hybrid" instrument combines conventional triple quadrupole functionality with ion trapping capabilities. Considering the merits of the LIT relative to the three-dimensional quadrupole ion trap, it is of interest to explore applications of ion/ion reactions in an LIT mass spectrometer. Recently, Hunt and coworkers $[27,28]$ reported ion/ion reactions in the Finnigan LTQ RF linear ion trap mass spectrometer (Thermo Electron Corporation, San Jose, CA), which is a stand-alone LIT instrument [22]. Secondary RF fields, imposed by superposition of RF 
voltages to the end lenses of the LIT, provided mutual storage of oppositely charged ions in the axial dimension, while the usual oscillating quadrupole field of the rod array provided mutual ion storage in the radial plane. Particular emphasis in that work was placed on electron transfer to polypeptide cations to effect dissociation. Several advantages associated with the use of the Finnigan LTQ setup for ion/ion reactions were also pointed out, such as axial segregation of precursor and reagent ions prior to reaction and soft injection of reagent ions.

Triple quadrupole instruments that allow for ion trapping offer a higher degree of flexibility than a single LIT mass spectrometer for conducting tandem-in-space, tandem-in-time, and combinations of tandem-in-time and tandem-in-space experiments. The potential dimensionality is increased when any of the rod arrays can serve as a reaction region and when ions can be readily transferred between quadrupoles. There are many possibilities to take advantage of the multiple choices of reaction region. For example, by use of the first quadrupole as an ion/ion reaction cell, the second as a collision cell, and the third as a mass analyzer, the beam-type fragmentation pattern of an ion/ion reaction product can be acquired. If the second quadrupole is used as an ion/ion reaction cell with the first and last quadrupoles acting as mass filters, the linked scanning functionality of the triple quadrupole could be used as a screening technique. The use of the third quadrupole as an ion/ion reaction region facilitates the study of the ion/ion reactions of either mass-selected parent ions from the first quadrupole or the beam-type fragments formed in the second quadrupole. In terms of reaction modes, there are, in principle, four modes to effect ion/ion reactions within a quadrupole rod array [29]. One involves continuously admitting ions of opposite polarity into the rod array without trapping either polarity. The likelihood for reactions in this mode is minimized by the fact that the number densities of the ions in a beam tend to be relatively low, the relative velocities of the ions are high, and it is difficult to obtain a high degree of spatial overlap of the ions. Two other modes involve storing one ion polarity while ions of the other polarity are continuously admitted into the rod array, which are denoted as positive ion transmission/ negative ion storage and negative ion transmission/ positive ion storage, respectively. Recently, the positive ion transmission/negative ion storage mode ion/ion reaction was implemented in the second quadrupole of a hybrid triple quadrupole/LIT instrument [29]. This mode is particularly useful in separation of ions of different mass and charge but very similar mass-tocharge ratio. The fourth mode, which is analogous to that used in most quadrupole ion trap studies [4], is the mutual storage mode. In this report, we describe mutual storage mode ion/ion reactions in the third quadrupole of a hybrid triple quadrupole/LIT instrument with axial trapping effected by applying unbalanced RF amplitudes to the two pairs of parallel rods in the quadrupole rod array.

\section{Experimental}

\section{Materials}

Peptides and proteins were purchased from Sigma (St. Louis, MO) and used without further purification. Electrospray solutions for the proteins and peptides were prepared in 50:49:1 methanol/water/acetic acid (vol/ $\mathrm{vol} / \mathrm{vol}$ ) to a final concentration of 5-10 $\mu \mathrm{M}$, unless otherwise specified. Perfluoro(methyldecalin) (PMD) was obtained from Aldrich (Milwaukee, WI).

\section{Mass Spectrometry}

All experiments were performed using a prototype version of a Q TRAP mass spectrometer [20] (Applied Biosystems/MDS SCIEX, Concord, Ontario, CN) modified for negative ion introduction from the side of Q3 by atmospheric sampling glow discharge ionization (ASGDI) [30], as discussed in detail elsewhere [29]. The ion path was based on that of a triple quadrupole mass spectrometer with the last quadrupole rod array (Q3) configured to operate either as a conventional RF/DC mass filter or as an LIT with mass-selective axial ejection (MSAE) [24]. The Q TRAP was operated at a drive RF of $650 \mathrm{kHz}$ with a nominal upper mass-to-charge limit of 4200 Th when the standard MSAE frequency was used. All experiments were controlled by MS Expo 1.75 software provided by Applied Biosystems/MDS SCIEX.

To create an axial RF barrier so that both ion polarities can be stored in three dimensions in the Q3 rod array, the RF amplitudes applied to the $\mathrm{X}$-poles and Y-poles of the Q3 rod array were unbalanced by subtracting some turns of the inductance coil from $X$-poles and adding those turns to the Y-poles. The degree of unbalance used in this study, which is defined as the percent deviation of the amplitude applied to a set of opposing rods from the average amplitude applied to both pairs of rods, was $54 \%$.

In a typical ion/ion reaction experiment, anions derived from PMD via ASGDI were radially injected into Q3 while the average RF amplitude was held at a value that yielded a low-mass cutoff, referred to as the "fill mass", of $m / z$ 160. During anion injection, the Q3 rod set was maintained at a DC potential between -10 $\mathrm{V}$ and $-20 \mathrm{~V}$, while a high trapping DC potential of $-200 \mathrm{~V}$ was applied to the containment lenses at each end of the Q3 rod array. The discharge was gated via software control so that it was on only during the anion accumulation period to avoid detector noise observed when the discharge was operated continuously. Several combinations of anion accumulation time were used to achieve the desired anion intensity. The anions were allowed to cool in Q3 for $50 \mathrm{~ms}$, during which time the Q3 fill mass was raised to $m / z 220$ and the DC potentials 
applied to the two end lenses of Q3 and the Q3 rods were adjusted to roughly the same DC potential level to prepare for cation injection. The ESI-generated cations were introduced axially into Q3 at relatively low kinetic energies and were cooled in Q3 for $20 \mathrm{~ms}$. During the subsequent mutual storage ion/ion reaction period, the Q3 fill mass was increased to $m / z$ 300. After a defined period of mutual storage, anions were ejected from Q3 either by applying attractive DC potentials to the containment lenses or by ramping the Q3 fill mass to $\mathrm{m} / \mathrm{z}$ 600. Just prior to the mass scan, the Q3 fill mass was dropped to $m / z 300$ and the DC voltage applied to the exit lens was adjusted to the optimum value for mass analysis, approximately $2-3 \mathrm{~V}$ repulsive with respect to the DC offset of the Q3 rod array. The positive ions trapped within the Q3 LIT after ion/ion reaction were subjected to MSAE using a supplementary AC signal at a frequency selected to give the desired mass range extension [31, 32]. The mass/charge ratios for the ion/ ion reaction spectra were calibrated from the various charge states of the known compounds. The spectra shown here were typically the average of 100-300 individual scans.

\section{Results and Discussion}

\section{Using Unbalanced RF for Mutual Storage in an LIT}

Conventional linear traps utilize DC potentials to provide axial ion containment. Consequently, only ions of one polarity can be trapped for arbitrarily long periods of time. To implement the mutual storage of oppositely charged ions, an additional RF field facilitates the containment of ions in the axial direction. Applying RF directly to the containment lenses of a quadruple is one of the solutions $[27,28]$. An alternative is to unbalance the RF amplitudes applied to the two sets of opposing poles of the quadrupole rod array. When the quadrupole and its containment lenses are all at the same DC level, subtracting a fraction of the RF level on one set of opposing poles and increasing the equivalent RF level on the other set of the opposing poles is equivalent to simply adding the same fraction of RF to the containment lenses. A conceptual depiction of this situation is shown in Figure 1. The degree of unbalance can then be controlled by adding and subtracting turns of the inductance coil in the RF circuitry to the X-and Y-poles, respectively (or vice versa).

The Q TRAP mass spectrometer [20] has a Q-q-Q $\mathrm{Q}_{\text {linear }}$ ion trap arrangement, in which each of the quadruple arrays can act as an LIT for mutual storage by unbalancing the RF amplitudes applied to the rod array. Given the location of the ASGDI source, which is attached on one side of the Q3 segment, it was most straightforward to study ion/ion reactions in Q3. Since the Q3 and Q2 rod arrays are capacitively coupled (the Q2 RF amplitude is approximately half that of the Q3 $\mathrm{RF})$, unbalancing the Q3 RF level also unbalances the

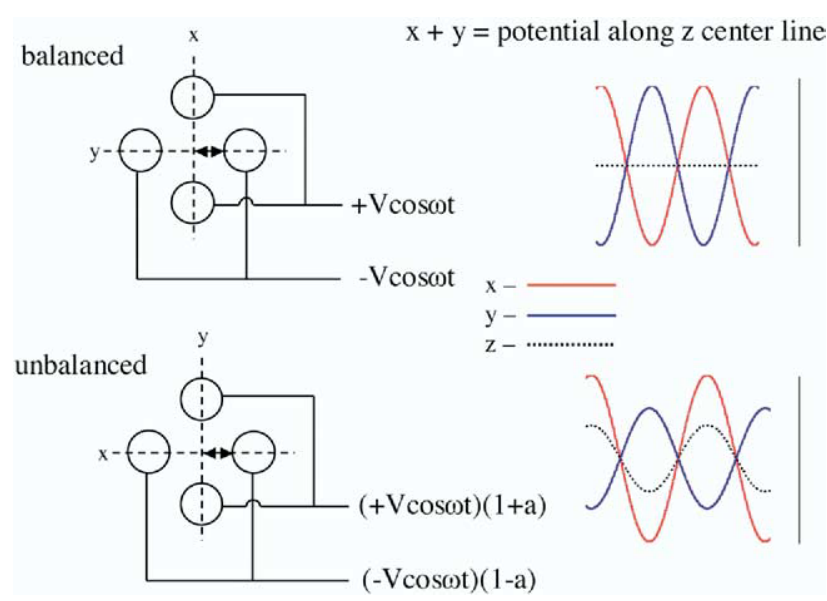

Figure 1. Conceptual depiction of unbalancing the RF amplitudes on the two sets of opposing poles of a quadrupole rod array. $\mathrm{V}$ is the RF amplitude applied on the rods, a is the degree of unbalance, and $\mathrm{x}, \mathrm{y}, \mathrm{z}$ represent the three dimensions.

Q2 RF level. It is of interest to study the mutual storage ion/ion reactions in Q2, as Q2 has a pressure about two orders of magnitude higher than that of Q3. However, transmitting anions from Q3 to Q2, while straightforward under balanced conditions [29], is complicated when both rod arrays are unbalanced. The unbalanced condition creates a barrier for transmission out of Q3 as well as into Q2. Presumably for this reason, transfer of anions from Q3 to Q2 was found to be highly inefficient. Therefore, all studies reported here involved mutual storage ion/ion reactions in Q3. A number of useful experiments are readily performed by use of Q3 as the ion/ion reactor. For example, using the Q3 quadrupole array as the ion/ion reaction cell is compatible with the use of Q1 under RF/DC mode for parent ion isolation, Q2 as a reaction region for beam-type collision-induced dissociation (CID), and Q3 as the ion/ion reactor and mass analyzer.

The Q3 quadrupole array was constructed from round gold-coated ceramic rods. There were two containment lenses, upstream and downstream of Q3, referred to as "IQ3" at the entrance to the array and the "exit lens" at the detector side of the array. For a typical mutual storage experiment, the rod offset of Q3 (RO3, a DC potential applied to all four rods of the array; same definition for RO1 and RO2), the DC potentials of IQ3, and the exit lens were almost the same. Therefore, the ions stored in Q3 are trapped solely by the usual RF field in the $x$ - and $y$-dimensions and, in the axial or z-dimension, by the RF introduced from unbalancing the RF amplitude of Q3. The degree of unbalance was an important factor in determining the mutual storage efficiency. The degree of unbalance could be either too high or too low. We found empirically that ion/ion reaction rates and storage efficiencies were highest when the unbalance was about 50\%. For example, thermalized cations and anions could each be stored for $5 \mathrm{~s}$ under identical conditions without significant ion 
loss. Therefore, all studies related here were conducted with Q3 operated with 54\% RF imbalance.

With the present ion source configuration, anions were radially injected into Q3. Under these conditions, the unbalanced Q3 RF condition alone was not sufficient to provide useful anion accumulation efficiencies. It was necessary to raise the DC potentials of the containment lenses, relative to that of the quadrupole array (Q3 lenses: IQ3 $=-200, \mathrm{RO3}=-20$, exit lens $=$ $-200 \mathrm{~V})$, during the anion accumulation period and for roughly $50 \mathrm{~ms}$ cooling step thereafter, to accumulate useful quantities of anions for subsequent reaction. After anion accumulation and cooling, the DC potentials could be reduced to approximately that of the Q3 rod offset while efficiently retaining $(>90 \%)$ the thermalized anions for several seconds. A rationale for this observation is that the radially injected anions pick up sufficient translational energy upon injection that they tend to execute relatively high amplitude motion and can easily penetrate the cone of reflection [24] and be accelerated toward the exit lenses by the strong net positive axial force. A relatively high repulsive potential is therefore needed to trap the anions until their motion is sufficiently damped via collisions to be retained with much lower trapping potentials. After anions were cooled, the potentials of IQ3 and exit lens were reduced roughly to the same potential as $\mathrm{RO} 3$ to allow for cation accumulation. Due to the high DC potentials needed to trap anions during injection, storing positive ions during anion injection was not feasible. Hence, anions were always accumulated first in these studies.

The electrospray-generated cations were directed axially along the normal flight path of the instrument at relatively low energies (several electronvolts). It was observed that the trapping efficiency was very sensitive to the axial kinetic energy of the positive ions. For example, when RO3 was $1 \mathrm{~V}$ more attractive for positive ions than RO2, 98\% cations were lost. When RO3 was $0.5 \mathrm{~V}$ more attractive, $60 \%$ of the cations were lost. Therefore, during cation injection, the DC levels of RO2 and $\mathrm{RO} 3$ were maintained at the same value. A trapping efficiency in excess of $90 \%$ was achieved with $5 \mathrm{~s}$ storage time under these conditions.

Both nitrogen and helium were evaluated as background gases for ion storage under the unbalanced RF condition. The gas enters the system via an inlet line connected to Q2. The limited gas conductance from Q2 gives rise to a roughly 100-fold difference in pressure between Q2 and Q3. Overall, helium was shown to lead to fewer ion losses over time scales ranging from $200 \mathrm{~ms}$ to $5 \mathrm{~s}$ when Q3 chamber pressure was kept roughly the same for these two gases. A maximum in cation signal strength was noted for positive ions at a Q3 pressure of $6 \times 10^{-5}$ torr. However, for radially injected anions, signal levels increased steadily as the Q3 pressure increased to the upper limit allowed by the vacuum protection system, i.e., $1 \times 10^{-4}$ torr. The maximum in cation signal is expected to be due to transmission losses as the cations pass through Q2 resulting from the
Table 1. Optimal conditions for mutual storage mode ion/ion reaction experiments in Q3

\begin{tabular}{ll}
\hline Parameter & \multicolumn{1}{c}{ Value } \\
\hline \hline P(O3) & $\begin{array}{l}7 \times 10^{-5} \text { torr } \\
\text { Helium } \\
\text { Q2 gas }\end{array}$ \\
$\begin{array}{l}\text { PMD pressure } \\
\text { Anion injection to O3 }\end{array}$ & $\mathrm{mT}$ Torr \\
Q3 fill mass & $\mathrm{m} 160$ \\
RO3 & $-10 \sim-25 \mathrm{~V}$ \\
IQ3, exit lens & $-200 \mathrm{~V}$ \\
Time & $600 \mathrm{~ms}$ \\
Cation injection & \\
Q3 fill mass & $\mathrm{m} / \mathrm{z} 220$ \\
RO0 - RO2 & $5 \mathrm{~V}$ \\
RO2, IQ3, RO3 & $-22 \mathrm{~V}$ \\
Exit lens & $-21.5 \mathrm{~V}$ \\
lon/lon reaction & \\
Q3 fill mass & $\mathrm{m} / \mathrm{z} \mathrm{300}$ \\
RO2, IQ3, RO3 & $-22 \mathrm{~V}$ \\
Exit lens & $-21.5 \mathrm{~V}$ \\
Time & variable \\
\hline
\end{tabular}

increased pressure. At Q3 pressures in excess of $6 \times$ $10^{-5}$ torr, Q2 pressures exceed 12 mTorr. Under such high Q2 pressures, positive ion transmission suffers due to scattering losses, poor extraction of cations from Q2, or a combination of both. The anions follow a different path into Q3 and therefore show a different Q3 pressure dependence.

\section{Mutual Storage Mode Ion/Ion Reactions in the Q3 LIT}

The ability to store thermalized positive and negative ions simultaneously in Q3 by utilizing the unbalanced $\mathrm{RF}$ enabled the mutual storage ion/ion reaction mode to be evaluated. A number of parametric studies were performed to optimize conditions for mutual storage ion/ion reactions in Q3. Table 1 summarizes the set of operating conditions that represents roughly optimal conditions for effecting ion/ion reactions in Q3 with the present instrumentation. Optimal conditions for ion/ ion reactions translate to the highest ion/ion reaction rates that can be achieved with minimal compromise in other key aspects of the overall experiment, such as sensitivity for analyte cations and mass resolution.

In considering the effects of experimental parameters on the observed ion/ion reaction rate, it is useful to examine fundamental parameters that affect ion/ion reaction rates under the conditions used here. In most analytical applications of ion/ion reactions, ions of one polarity represent the analyte while the oppositely charged ions serve as reagents intended to transform the analyte ions in some way, such as changing the charge state(s) of the analyte ions. In this scenario, it is desirable to use an excess of reagent ions to maximize the reaction rate. When there is a great excess of reagent ions available for reaction, pseudo-first order kinetics are observed. For the case of proton transfer, the relationship is: 


$$
\left[M H_{(\mathrm{Z}-1)}^{(\mathrm{Z}-1)+}\right]_{t}=\left[\mathrm{MH}_{\mathrm{Z}}^{\mathrm{Z}}\right]_{0} e^{-n k t}
$$

where $Z$ is the unit charges of the ions, $n$ is the "effective" number density of the reagent anions, $k$ is the rate constant for the proton transfer reaction, and $t$ is the reaction time. The term "effective" is used for reagent anion number density to account for the fact that $\mathrm{n}$ is not constant throughout the entire trapping volume and the degree of spatial overlap between the oppositely charged ion populations may vary with experimental conditions. The extent of ion/ion reaction in these studies, as reflected in the conversion of positively charged reactant ions to positively charged products, increased monotonically with anion accumulation time over periods extending to as high as $600 \mathrm{~ms}$. This suggests that the reagent anion storage capacity of Q3 was not reached under the conditions of this study. Had the storage capacity been reached, the ion/ion reaction rate would be expected to become independent of anion accumulation time, as the effective anion density reaches a maximum. Anion accumulation times sufficient to ensure that the extent of ion/ion reaction increase monotonically with time were used throughout these studies. This condition indicates the presence of excess reagent ions, as is necessary for eq 1 to hold.

Previous studies have suggested that the formation of a stable ion/ion reaction complex is the rate-determining event for an ion/ion reaction in an electrodynamic ion trap [33]. The maximum rate constant for this process is given by:

$$
k_{\max }=\frac{4 \pi Z_{1}^{2} Z_{2}^{2} e^{4}}{\left(4 \pi \varepsilon_{0}\right) \mu^{2} v_{\text {rel }}^{3}}
$$

where $Z_{1}$ and $Z_{2}$ are the unit charges of the oppositely charged ions, $e$ is the elementary charge, $\varepsilon_{0}$ is the permittivity of vacuum, $\mu$ is the reduced mass of the ion/ion pair, and $\nu_{\text {rel }}$ is the relative velocity of the collision pair. No quantitative data are available to assess how closely $\mathrm{k}_{\max }$ approximates the actual rate constant, $\mathrm{k}$, in the ion trap environment. The relationship for $k_{\max }$ assumes that once the reactants reach a critical distance of separation that some means for removal of small fraction of the relative velocity is active [33]. This may involve collision with a third body, such as a background gas atom or molecule [34], or a so-called tidal effect $[35,36]$. If third body collisions are important and $\mathrm{k}<\mathrm{k}_{\max }$, increasing bath gas pressure would be expected to lead to an increase in $\mathrm{k}$ until $\mathrm{k}=$ $\mathrm{k}_{\max }$. This effect, however, is difficult to distinguish from other means by which background gas pressure can affect reaction rates. For example, eq 2 indicates that, for a given reactant pair, the relative velocity of the reactants is a parameter in the determination of $k_{\max }$ that might be affected by experimental conditions. Ion/neutral collisions tend to thermalize the ions, thereby affecting $\mathrm{v}_{\text {rel }}$. Hence, this factor alone leads to the expectation that background gas pressure should affect reaction rates, at least up to the point where thermalization of the ions is sufficiently rapid so that further increases in pressure have little effect. The reaction rate is measured directly rather than the reaction rate constant. The maximum reaction rate of the analyte cation, $\mathrm{R}_{\max }$ under these pseudo first order conditions is the product of the effective number density and the maximum rate constant:

$$
R_{\max }=n k_{\max } .
$$

The effective number density of the reagent ions is determined both by the number of reagent ions and by the degree of spatial overlap. Bath gas pressure can affect both of these parameters. Ion injection efficiency into an ion trap, particularly radial injection, is bath gas pressure dependent. Hence the number of reagent anions can be affected by bath gas pressure. Spatial overlap of the oppositely charged ion populations can also be affected by background pressure via its collisional cooling role that tends to concentrate ions at the center of the ion trap. For the reasons just given, increases in ion/ion reaction rates with increasing bath gas pressure are likely to arise from a combination of effects.

It is clear from eqs 1-3 that, in the case of excess reagent ions, experimental conditions that affect the reaction rate for a given ion/ion reaction pair do so through their effects on the effective number density and rate constant of the reaction. However, the detailed mechanism by which a particular experimental parameter affects reaction rates is not always clear when several factors can be influenced by a change in experimental conditions. The detailed role of background gas, mentioned above, is a case in point. Presumably for the reasons mentioned above, ion/ion reaction rates showed an increase with Q3 pressure up to the maximum pressure accommodated by the vacuum protection system (i.e., $10^{-4}$ torr). Most quadrupole ion trap studies have been performed using helium as the major background gas at a pressure of roughly $10^{-3}$ torr and reaction rates are higher at $10^{-3}$ torr than at $10^{-4}$ torr in the quadrupole ion trap. Therefore, it is expected that higher background pressures in the LIT will lead to greater reaction rates. We noted that, in analogy with the quadrupole ion trap, use of helium as buffer gas led to greater reaction rates than nitrogen at similar pressures. Bath gas identity, like bath gas pressure, can affect several parameters so that it is difficult to clearly identify why helium was more effective than the heavier nitrogen bath gas. For example, bath gas identity is a factor in anion injection efficiency, thereby affecting the anion density, and may also affect relative velocity. The heavier nitrogen bath gas is expected to give rise to a greater degree of scattering, particularly for the reagent anions.

In analogy with the quadrupole ion trap, the amplitude of the RF applied to the LIT rod array, which is proportional to the low mass cutoff, determines the pseudo potential well depth for trapping both polarities 


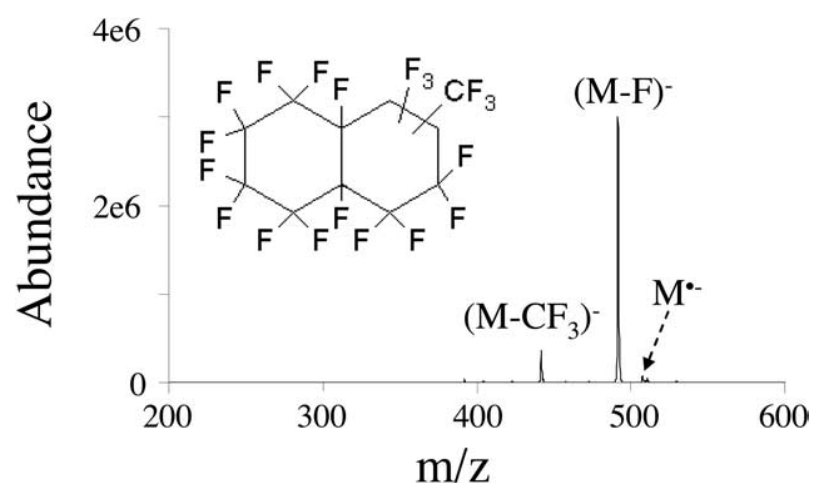

Figure 2. Mass spectrum of PMD by radial injection from glow discharge source. Q3 fill mass was 160 during injection and 300 for cooling step.

of ions and is a key factor in determining the extent of ion/ion reaction. The RF amplitude determines the $\mathrm{m} / \mathrm{z}$ range over which ions of opposite polarity can be stored simultaneously [37]. The RF amplitude establishes both the upper and lower $\mathrm{m} / \mathrm{z}$ limits to ion storage and there is a trade-off between them. Under the $54 \%$ Q3 unbalanced condition, we found that anions derived from glow discharge ionization of perfluoro(methyldecalin) $(\mathrm{PMD}$, molecular mass $=512 \mathrm{Da})$ were more effective in reducing high mass protein cations to low charge states than those derived from the commonly used reagent perfluoro-1,3-dimethylcyclohexane $(\mathrm{PDCH}$, molecular mass $=400 \mathrm{Da}$ ). A higher RF level could be used with the PMD anions than with the PDCH anions, which resulted in a higher upper $\mathrm{m} / \mathrm{z}$ limit for storage of high mass product ions (see below). Figure 2 shows the mass spectrum of PMD anions resulting from radial injection into Q3 at a fill mass of $m / z 160$ during anion injection and $m / z 300$ during the cooling step. If PMD behaves analogously to $\mathrm{PDCH}$, glow discharge ionization yields predominantly the radical anion and the fragment ions are formed as a result of the relatively violent process associated with radial injection into the ion trap [38]. Since the optimum low mass cutoff value for ion/ion reaction was between three hundred and four hundred depending on the $\mathrm{m} / \mathrm{z}$ values of the positive ions, $(\mathrm{M}-\mathrm{F})^{-}(\mathrm{m} / \mathrm{z} 493)$ and $\left(\mathrm{M}^{-} \mathrm{CF}_{3}\right)^{-}(\mathrm{m} / \mathrm{z} 443)$ were the major anions involved in the ion/ion reactions. The lower mass anions, such as $\left(\mathrm{M}^{-} \mathrm{C}_{5} \mathrm{~F}_{9}\right)^{-}(\mathrm{m} / \mathrm{z} 281),\left(\mathrm{M}-\mathrm{C}_{4} \mathrm{~F}_{9}\right)^{-}$ $(\mathrm{m} / \mathrm{z} 293)$ and $\left(\mathrm{M}-\mathrm{C}_{3} \mathrm{~F}_{7}\right)^{-}(\mathrm{m} / \mathrm{z} 331)$, were ejected prior to the mutual storage period.

Mutual storage of multiply charged protein ions with an excess of PMD anions allowed for the charge state reduction of the protein ions to singly and doubly charged ions via sequential proton transfer reactions in Q3 operated as an LIT. Figure 3 shows the post-ion/ion mass spectra of (Figure 3a) bovine ubiquitin, (Figure 3b) horse heart myoglobin, and (Figure 3c) bovine serum albumin (BSA) acquired with axial ejection frequencies of 80,42 , and $10 \mathrm{kHz}$, respectively. Approximate values for the resolving powers associated with the three various mass analysis conditions, as determined by full width at half maximum for the expanded peaks are 200, 180 , and 100, respectively. A decrease in resolution with decreasing axial ejection frequency has also been noted with resonance ejection from quadrupole ion traps. However, the absolute resolution values observed here are poorer than those recently reported with a quadrupole ion trap over comparable ranges of $m / z$ [39]. It is premature, however, to draw conclusions about mass analysis characteristics of MSAE at high $\mathrm{m} / \mathrm{z}$ values, particularly under conditions as highly unbalanced as those used here. The performance of the LIT as an analyzer for high $\mathrm{m} / \mathrm{z}$ ions will receive further attention now that it is possible to readily form such ions via ion/ion reactions. The upper $\mathrm{m} / \mathrm{z}$ limit for product ions is also of interest. Unambiguous evidence for signals for the +1 charge state of BSA was not observed under the conditions used here, although clear signals due to the +2 and +3 charge states were noted, as shown in Figure $3 c$. The relative abundances of these signals indicate that +1 ions should also have been formed at this reaction time. Therefore, this observation suggests that the effective upper mass-to-charge ratio limit of the system with the current configuration is between 33,000 and 66,000 . This limit is likely to be imposed by the ability to store both ions in excess of $\mathrm{m} / \mathrm{z} 33,000$ and $\mathrm{m} / \mathrm{z}$ 493 simultaneously. However, ion detection and ion ejection issues may also play a role in poor detection of ions in excess of $m / z 33,000$.

The data of Figure 3 clearly demonstrate that it is

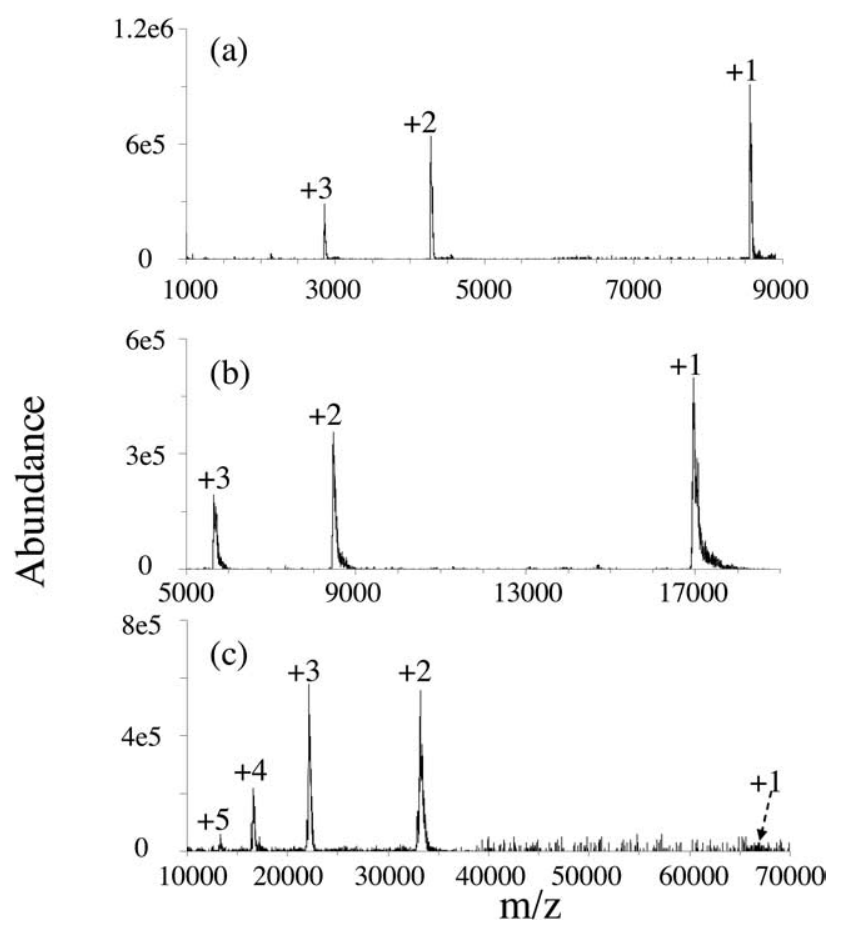

Figure 3. Post-ion/ion reaction mass spectra of (a) bovine ubiquitin, (b) horse heart myoglobin, and (c) bovine serum albumin (BSA) acquired after mutual storage of $600 \mathrm{~ms}, 600 \mathrm{~ms}$, and $1 \mathrm{~s}$ with an axial ejection frequency of 80,42 , and $10 \mathrm{KHz}$, respectively. 


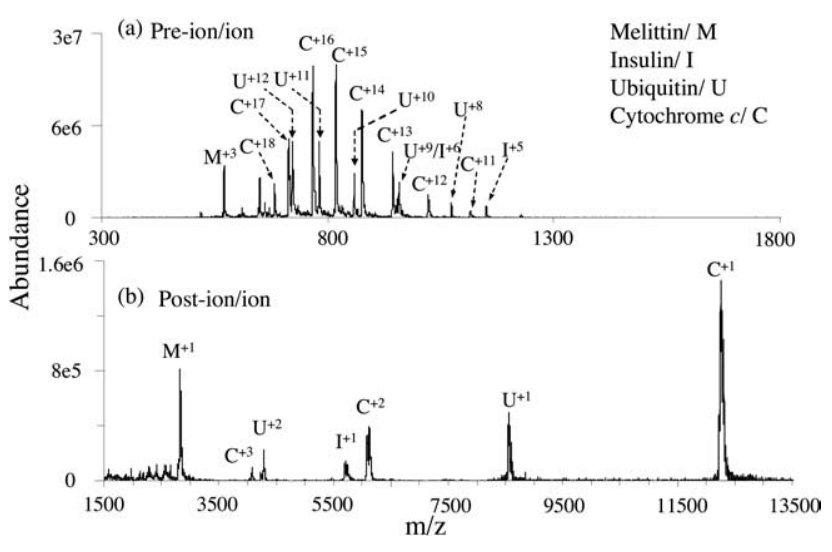

Figure 4. (a) Mass spectrum of the four-component mixture containing melittin, insulin, ubiquitin, and cytochrome $c$, and (b) post-ion/ion reaction spectrum of the mixture with anion accumulation and ion/ion mutual storage times being $510 \mathrm{~ms}$ and 300 $\mathrm{ms}$

possible to reduce the charges of multiply charged proteins as large as roughly $30 \mathrm{kDa}$ to arbitrarily low values via ion/ion proton transfer reactions in the Q3 LIT. An analytical application demonstrated with the quadrupole ion trap that takes advantage of this capability is its use in reducing complications arising from the electrospray ionization of protein mixtures [5-7]. This capability is illustrated for the LIT using mutual storage mode ion/ion reactions involving cations from a four-component mixture containing melittin, insulin, ubiquitin and cytochrome $c$. The pre-ion/ion reaction electrospray mass spectrum of this mixture is shown in Figure 4a while the post-ion/ion reaction mass spectrum is shown in Figure $4 \mathrm{~b}$. The latter spectrum was collected following a mutual storage period of $300 \mathrm{~ms}$ using an ejection frequency of $60 \mathrm{kHz}$.

The use of ion/ion proton transfer reactions for manipulating precursor and product ion charge states facilitates greatly the study of whole protein ions in the quadrupole ion trap $[4,6,7,10-12,14-19,39-48]$. A key experiment in studying the charge state dependent fragmentation behavior of whole protein ions in the quadrupole ion trap is to apply ion/ion proton transfer reactions to product ions formed via in-trap collisional activation. By converting all product ions largely to the +1 charge state, many possible charge state ambiguities associated with the initially formed product ions are avoided and the relative contributions of the various dissociation channels can be evaluated without considering charge state dependent ion detection efficiency. Virtually all studies reported to date that apply ion/ion proton transfer reactions to product ions have employed ion trap collisional activation as the means for generating fragments. In-trap collisional activation is also possible in the Q3 LIT [26]. However, the hybrid triple quadrupole linear ion trap configuration of the current instrument also allows for the use of beam-type collisional activation as the means of generating product ions. To illustrate this new variation in acquiring post-ion/ion reaction product ion spectra, the individual $[\mathrm{M}+8 \mathrm{H}]^{8+}$ (electrosprayed from 50:50 methanol/ water solution), $[\mathrm{M}+12 \mathrm{H}]^{12+}$ parent ions and a range of parent ion charge states of ubiquitin $\left([\mathrm{M}+10 \mathrm{H}]^{10+} \sim\right.$ $[\mathrm{M}+13 \mathrm{H}]^{13+}$ ) were subjected to beam-type collisional activation in Q2 and the product ions collected in Q3 were subjected to ion/ion proton transfer reactions to yield largely singly-charged products. In each case, the final post-ion/ion product ion spectrum was collected via MSAE from Q3 at a resonance ejection frequency of $80 \mathrm{kHz}$. Figure 5 shows pre- and post-ion/ion product ion spectra arising from beam-type CID of the +8 charge state of ubiquitin (laboratory collision energy $=$ $480 \mathrm{eV}$ ). The post-ion/ion reaction product ion spectrum was acquired after subjecting the product ions to reactions with anions derived from PMD for $550 \mathrm{~ms}$. The product ions in the post-ion/ion reaction spectrum could be readily assigned as either b- or y-type product ions via comparison with the expected product ion masses of ubiquitin. Evidence for fragmentation of a total of 35 out of 76 of the protein backbone amide bonds (46\%) was identified from beam-type CID of this charge state. Similar fragmentation channels and sequence coverage were noted with ion trap collisional activation of this charge state [15]. The higher charge state, +12 , yielded fewer fragments (26 out of 76 ) than the +8 charge state under beam-type fragmentation condition, which is consistent with the behavior observed previously in the quadrupole ion trap when the in-trap fragmentation was evaluated [15]. When a range of charge states of ubiquitin ions $\left([\mathrm{M}+10 \mathrm{H}]^{10+} \sim[\mathrm{M}+\right.$ $13 \mathrm{H}]^{13+}$ ) were subjected to beam-type fragmentation, 38 backbone cleavages were identified (data not shown), which illustrates the potential desirability of fragmenting several charge states at the same time. Generally, similar fragmentation patterns were seen with beamtype CID in the Q TRAP as in the in-trap CID of the same charge states, ranging from $[\mathrm{M}+12 \mathrm{H}]^{12+}$ to $[\mathrm{M}+$ $7 \mathrm{H}]^{7+}$, which were acquired in quadruple ion traps. However, the beam-type CID experiment does not require a frequency tuning step, as does an in-trap CID experiment, and it is readily applied to ranges of charge states simultaneously.

Another important experimental tool for an ion trapping system capable of effecting ion/ion reactions is the ability to inhibit selectively the rate of ion/ion reactions. This capability has been illustrated with the quadrupole ion trap [10] and is referred as "ion parking". It can be accomplished, for example, via the resonance excitation of a particular ion during the period in which ions of opposite polarities are stored simultaneously. The ion/ion reaction rate for the accelerated ion is reduced greatly due to both a decrease in the spatial overlap and an increase in the relative velocity of the oppositely-charged ion reactants (i.e., the effective number density is reduced as is the rate constant). An important application of ion parking is gas-phase concentration and purification [45], and the formation of ions that cannot be directly produced by 

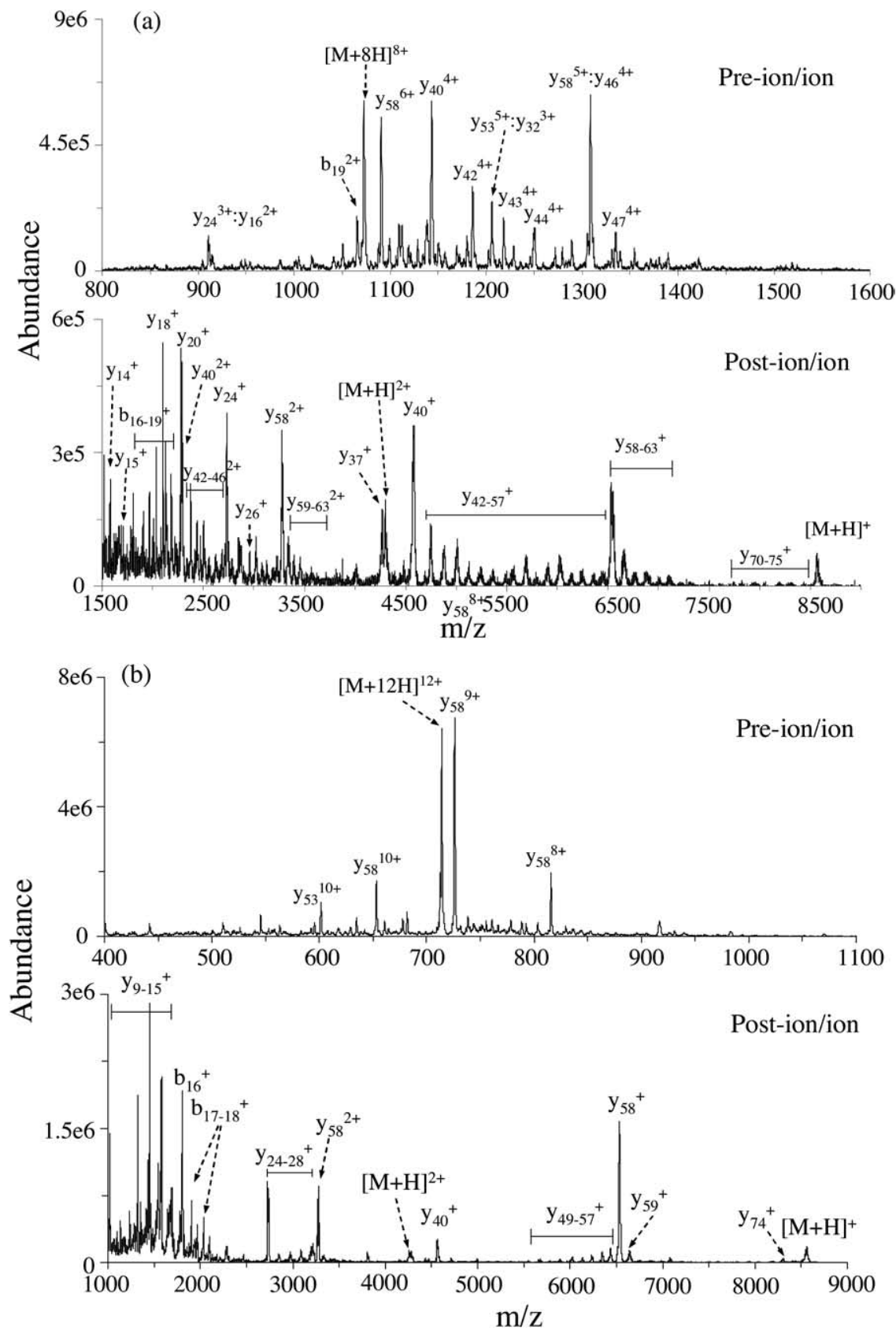

Figure 5. Beam-type CID MS/MS spectrum and the corresponding post-ion/ion reaction MS/MS spectrum of (a) the $[\mathrm{M}+8 \mathrm{H}]^{8+}$ ion of ubiquitin at collision energy of $480 \mathrm{eV}$, and $(\mathbf{b})[\mathrm{M}+12 \mathrm{H}]^{12+}$ ion of ubiquitin at a collision energy of $396 \mathrm{eV}$. The post-ion/ion MS/MS spectrum was acquired at an axial ejection frequency of $80 \mathrm{KHz}$.

ESI. The ion parking experiment was conducted in the Q3 LIT and is illustrated here with bovine cytochrome c. Effective ion parking experiments have been performed for charge states of cytochrome $c$ from +8 to +14 . In all cases, significant concentration of signal in the ion for which the resonance excitation of signal was most closely tuned was observed. The experiments summarized in Figure 6 may be used to obtain a semi-quantitative estimate of the efficiency of the ion parking procedure, defined as the fraction of the initial reactant ion population that can be accumulated in a specific charge state via the ion parking procedure.

Figure 6a shows the pre-ion/ion electrospray mass spectrum of cytochrome $c$, Figure $6 \mathrm{~b}$ shows the postion/ion mass spectrum (no ion parking) after a 100-ms anion accumulation period and a 300-ms mutual storage time period, and Figure 6c shows the results using the same ion/ion reaction conditions with a resonance excitation signal of $64.1 \mathrm{kHz}, 1.1 \mathrm{~V}_{p-p}$. The efficiency of ion parking experiment was estimated by assuming a 


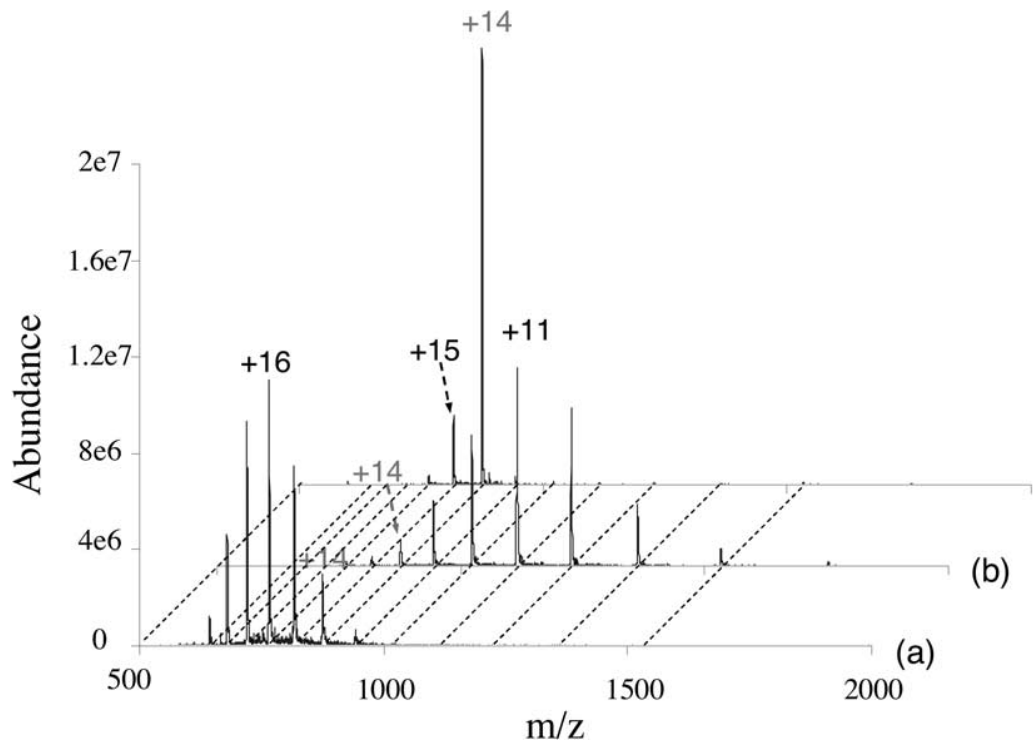

Figure 6. Mass spectra of bovine cytochrome $c$ ions acquired in (a) pre-ion/ion, (b) post-ion/ion, and (c) ion parking modes $\left(64.1 \mathrm{kHz}, 1.1 \mathrm{~V}_{p-p}\right)$ using a resonance ejection frequency of $60 \mathrm{kHz}$. The anion injection and mutual storage periods used in (b) and (c) were $100 \mathrm{~ms}$ and $300 \mathrm{~ms}$ respectively.

linear dependence of the detector response to the charge state of the ion. After normalizing ion abundances according to charge state, roughly $60 \%$ of the ions of Figure $6 \mathrm{a}$ are concentrated in the +14 charge state after ion parking (Figure 6c).

The efficiency of the ion parking experiment associated with Figure 6 is somewhat lower than has been observed with quadrupole ion traps [10]. Furthermore, the time dependence of the parking experiment showed that the ion/ion reaction rate of the +15 charge state, and the +16 charge state to a lesser extent, were affected (reduced) when the maximum rate reduction for the +14 ions was achieved. This suggests that the higher charge state ions were undergoing a degree of ion acceleration resulting in the inhibition of their reaction rates. The frequency dependence of the ion parking experiment using dipolar resonance excitation in the Q3 LIT is shown in Figure 7. A resonance excitation amplitude of $1.1 \mathrm{~V}_{p-p}$ was stepped at $100 \mathrm{~Hz}$ increments across the +14 charge state of cytochrome $c$ during the ion/ion

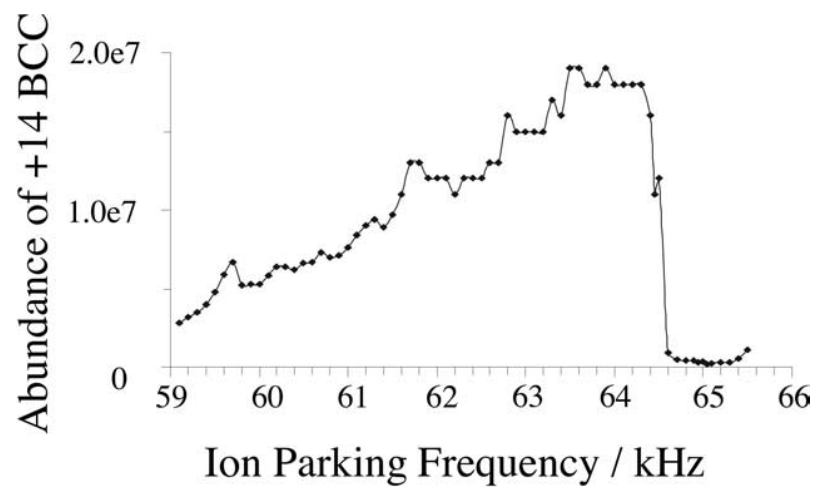

Figure 7. Ion parking absorption profile of the $[\mathrm{M}+14 \mathrm{H}]^{14+}$ ion of bovine cytochrome $c$. reaction period. Ion parking with relatively high efficiency was found using resonance excitation voltages at frequencies on the high frequency side, which is around $64.2 \mathrm{kHz}$. Higher charge states such as +15 and +16 were also observed along with the parked charge state with very low signals, if any, for lower charge states. The parking efficiency decreased slowly as the frequency was reduced with a concomitant increase in signals arising from lower charge states. The asymmetric shape of the ion parking absorption profile is highly suggestive of the presence of higher order multi-pole fields, which are known to give rise to asymmetric resonance absorption profiles in quadrupole ion traps [42-44]. In this case, higher order fields would be expected to arise from the use of round quadrupole rods [22]. It is not clear, at this point, if the use of unbalanced RF might also contribute to this effect. Future work will employ the use of RF voltages applied directly to the containment lenses along with more balanced RF to determine the extent to which unbalanced rods lead to compromises in mass resolution and ion parking resolution.

\section{Conclusions}

A hybrid triple quadrupole/linear ion trap mass spectrometer has been modified to enable experiments that involve ion/ion proton transfer reactions via mutual storage of both ion polarities. Both positive and negative ions can be stored simultaneously by unbalancing the RF amplitude applied to the two sets of opposing poles of the Q3 rod array. Essentially all of the key ion/ion proton transfer reaction experiments already demonstrated to be useful in complex protein mixture analysis scenarios using quadrupole ion traps have 
been demonstrated here with the modified hybrid instrument. These experiments include the use of ion/ion reactions applied to electrospray of protein mixtures, ion/ion reactions applied to product ion mixtures to facilitate product ion assignment, and ion parking experiments for protein ion concentration. Product ions can be generated with this instrument either via in-trap collisional activation, in analogy with convention ion trap collisional activation, or via beam-type collisional activation. The latter mode was used to generate product ions in this work. Under the unbalanced Q3 RF condition used to collect the data reported here, the system provides an upper mass-to-charge ratio limit of at least 33,000, with mass resolving power of several hundred at high $\mathrm{m} / \mathrm{z}$. Future studies will be directed to the optimization of mass analysis characteristics as well as the development of novel ion/ion reaction experiments in the hybrid instrument. The extent to which the use of unbalanced RF for ion trapping adversely affects other performance characteristics will be explored by directly applying RF to the containment lenses of the Q3 rod array only during the mutual ion storage periods while employing more balanced RF voltages to the rod array. We will also examine mutual ion storage in other rod arrays in the hybrid instrument.

\section{Acknowledgments}

The work at Purdue was sponsored by MDS SCIEX, an Industrial Associate of the Department of Chemistry. The authors acknowledge Adam Lau of MDS SCIEX for providing custom instrument control software. They thank Randy Replogle and Greg Hawkins for construction of the ASGDI source components.

\section{References}

1. Mather, R. E.; Todd, J. F. J. The Quadrupole Ion Store (Quistor). 7. Simultaneous positive-negative ion mass-spectrometry. Int. J. Mass Spectrom. Ion Phys. 1980, 33, 159-165.

2. Louris, J. N.; Brodbelt-Lustig, J. S.; Cooks, R. G.; Glish, G. L.; Van Berkel, G. J.; McLuckey, S. A. Ion isolation and sequential stages of mass-spectrometry in a quadrupole ion trap massspectrometer. Int. J. Mass Spectrom. Ion Processes 1990, 96, 117-137.

3. McLuckey, S. A.; Glish, G. L.; Van Berkel, G. J. Multiple stages of mass-spectrometry in a quadrupole ion trap mass-spectrometer-prerequisites. Int. J. Mass Spectrom. Ion Process. 1991, 106, 213-235.

4. McLuckey, S. A.; Stephenson, J. L. Jr., Ion/ion chemistry of high-mass multiply charged ions. Mass Spectrom. Rev. 1998, 17, 369-407.

5. Stephenson, J. L. Jr.; McLuckey, S. A. Charge manipulation for improved mass determination of high-mass species and mixture components by electrospray mass spectrometry. J. Mass Spectrom. 1998, 33, 664-672.

6. McLuckey, S. A.; Stephenson, J. L. Jr.; Asano, K. G. Ion/ion proton-transfer kinetics: Implications for analysis of ions derived from electrospray of protein mixtures. Anal. Chem. 1998, 70, 1198-1202.

7. Stephenson, J. L. Jr.; McLuckey, S. A. Ion/ion proton transfer reactions for protein mixture analysis. Anal. Chem. 1996, 68, $4026-4032$.
8. Pan, P.; McLuckey, S. A. Electrospray ionization of protein mixtures at low pH. Anal. Chem. 2003, 75, 1491-1499.

9. McLuckey, S. A.; Wu, J.; Bundy, J. L.; Stephenson, J. L. Jr.; Hurst, G. B. Oligonucleotide mixture, analysis via electrospray and ion/ion reactions in a quadrupole ion trap. Anal. Chem. 2002, 74, 976-984.

10. McLuckey, S. A.; Reid, G. E.; Wells, J. M. Ion parking during ion/ion reactions in electrodynamic ion traps. Anal. Chem. 2002, 74, 336-346.

11. Reid, G. E.; Shang, H.; Hogan, J. M.; Lee, G. U.; McLuckey, S. A. Gas-phase concentration, purification, and identification of whole proteins from complex mixtures. J. Am. Chem. Soc. 2002, 124, 7353-7362.

12. Amunugama, R.; Hogan, J. M.; Newton, K. A.; McLuckey, S. A. Whole protein dissociation in a quadrupole ion trap: Identification of an a priori unknown modified protein. Anal. Chem. 2004, 76, 720-727.

13. Stephenson, J. L. Jr.; McLuckey, S. A. Ion/ion reactions in the gas phase: Proton transfer reactions involving multiplycharged proteins. J. Am. Chem. Soc. 1996, 118, 7390-7397.

14. Wells, J. M.; Stephenson, J. L. Jr.; McLuckey, S. A. Charge dependence of protonated insulin decompositions. Int. J. Mass Spectrom. 2000, 203, A1-A9.

15. Reid, G. E.; Wu, J.; Chrisman, P. A.; Wells, J. M.; McLuckey, S. A. Charge-state-dependent sequence analysis of protonated ubiquitin ions via ion trap tandem mass spectrometry. Anal. Chem. 2001, 73, 3274-3281.

16. Newton, K. A.; Chrisman, P. A.; Reid, G. E.; Wells, J. M.; McLuckey, S. A. Gaseous apomyoblobin ion dissociation in a quadrupole ion trap: $[\mathrm{M}+2 \mathrm{H}](2+)-[\mathrm{M}+21 \mathrm{H}](21+)$. Int. J. Mass Spectrom. 2001, 212, 359-376.

17. Engel, B. J.; Pan, P.; Reid, G. E.; Wells, J. M.; McLuckey, S. A. Charge state dependent fragmentation of gaseous protein ions in a quadrupole ion trap: Bovine ferri-, ferro-, and apocytochrome c. Int. J. Mass Spectrom. 2002, 219, 171-187.

18. Chrisman, P. A.; McLuckey, S. A. Dissociations of disulfidelinked gaseous polypeptide/protein anions: Ion chemistry with implications for protein identification and characterization. J. Proteome Res. 2002, 1, 549-557.

19. Hogan, J. M.; McLuckey, S. A. Charge state dependent collision-induced dissociation of native and reduced porcine elastase. J. Mass Spectrom. 2003, 38, 245-256.

20. Hager, J. W. A new linear ion trap mass spectrometer. Rapid Commun. Mass Spectrom. 2002, 16, 512-526.

21. Quarmby, S. T.; Yost, R. A. Fundamental studies of ion injection and trapping of electrosprayed ions on a quadrupole ion trap. Int. J. Mass Spectrom. 1999, 191, 81-102.

22. Schwartz, J. C.; Senko, M. W.; Syka, J. E. P. A two-dimensional quadrupole ion trap mass spectrometer. J. Am. Soc. Mass Spectrom. 2002, 13, 659-669.

23. Church, D. A. Storage-ring ion trap derived from the linear quadrupole radio-frequency mass filter. J. Appl. Phys. 1969, 40, 3127-3134.

24. Londry, F. A.; Hager, J. W. Mass selective axial ion ejection from a linear quadrupole ion trap. J. Am. Soc. Mass Spectrom. 2003, 14, 1130-1147.

25. Hager, J. W.; Le Blanc, J. C. Y. Product ion scanning using a Q-q-Q (linear ion trap) [Q TRAP (TM)] mass spectrometer. Rapid Commun. Mass Spectrom. 2003, 17, 1056-1064.

26. Collings, B. A.; Stott, W. R.; Londry, F. A. Resonant excitation in a low-pressure linear ion trap. J. Am. Soc. Mass Spectrom. 2003, 14, 622-634.

27. Syka, J. E. P.; Coon, J. J.; Schroeder, M. J.; Shabanowitz, J.; Hunt, D. F. Peptide and protein sequence analysis by electron transfer dissociation mass spectrometry. Proc. Natl. Acad. Sci. U.S.A. 2004, 101, 9528-9533. 
28. Coon, J. J.; Syka, J. E. P.; Schwartz, J. C.; Shabanowitz, J.; Hunt, D. F. Anion dependence in the partitioning between proton and electron transfer in ion/ion reactions. Int. J. Mass Spectrom. 2004, 236, 33-42.

29. Wu, J.; Hager, J. W.; Xia, Y.; Londry, F. A.; McLuckey, S. A. Positive ion transmission mode ion/ion reactions in a hybrid linear ion trap. Anal. Chem. 2004, 76, 5006-5015.

30. McLuckey, S. A.; Glish, G. L.; Asano, K. G.; Grant, B. C. Atmospheric sampling glow-discharge ionization source for the determination of trace organic-compounds in ambient air. Anal. Chem. 1988, 60, 2220-2227.

31. Kaiser, R. E. Jr.; Cooks, R. G.; Moss, J.; Hemberger, P. H. Mass range extension in a quadrupole ion trap mass spectrometer. Rapid Commun. Mass Spectrom. 1989, 3, 50-53.

32. Kaiser, R. E. Jr.; Cooks, R. G.; Stafford, G. C.; Syka, J. E. P.; Hemberger, P. H. Operation of a quadrupole ion trap massspectrometer to achieve high mass charge ratios. Int. J. Mass Spectrom. Ion Processes 1991, 106, 79-115.

33. Wells, J. M.; Chrisman, P. A.; McLuckey, S. A. Formation and characterization of protein-protein complexes in vacuo. J. Am. Chem. Soc. 2003, 125, 7238-7249.

34. Thomson, J. J. Philos. Mag. 1924, 47, 337-378.

35. Bates, D. R.; Morgan, W. L. New recombination mechanismTidal termolecular ionic recombination. Phys. Rev. Lett. 1990, 64, 2258-2260.

36. Morgan, W. L.; Bates, D. R. Tidal termolecular ionic recombination. J. Phys. B 1992, 25, 5421-5430.

37. Stephenson, J. L. Jr.; McLuckey, S. A. Anion effects on storage and resonance ejection of high mass-to-charge cations in quadrupole ion trap mass spectrometry. Anal. Chem. 1997, 69, 3760-3766.

38. Stephenson, J. L. Jr.; McLuckey, S. A. Adaptation of the Paul Trap for study of the reaction of multiply charged cations with singly charged anions. Int. J. Mass Spectrom. Ion Process. 1997, $162,89-106$.
39. Reid, G. E.; Wells, J. M.; Badman, E. R.; McLuckey, S. A Performance of a quadrupole ion trap mass spectrometer adapted for ion/ion reaction studies. Int. J. Mass Spectrom. 2003, 222, 243-258.

40. Stephenson, J. L. Jr.; McLuckey, S. A. Simplification of product ion spectra derived from multiply charged parent ions via ion/ion chemistry. Anal. Chem. 1998, 70, 3533-3544.

41. Schaaff, T. G.; Cargile, B. J.; Stephenson, J. L. Jr.; McLuckey, S. A. Ion trap collisional activation of the $(\mathrm{M}+2 \mathrm{H})(2+)-(\mathrm{M}$ $+17 \mathrm{H})(17+)$ ions of human hemoglobin beta-chain. Anal. Chem. 2000, 72, 899-907.

42. Cargile, B. J.; McLuckey, S. A.; Stephenson, J. L. Jr. Identification of bacteriophage MS2 coat protein from E-coli lysates via ion trap collisional activation of intact protein ions. Anal. Chem. 2001, 73, 1277-1285.

43. Chrisman, P. A.; Newton, K. A.; Reid, G. E.; Wells, J. M.; McLuckey, S. A. Loss of charged versus neutral heme from gaseous holomyoglobin ions. Rapid Commun. Mass Spectrom. 2001, 15, 2334-2340.

44. He, M.; Reid, G. E.; Shang, H.; Lee, G. U.; McLuckey, S. A. Dissociation of multiple protein ion charge states following a single gas-phase purification and concentration procedure. Anal. Chem. 2002, 74, 4653-4661.

45. Reid, G. E.; McLuckey, S. A. "Top down" protein characterization via tandem mass spectrometry. J. Mass Spectrom. 2002, $37,663-675$.

46. Stephenson, J. L. Jr.; McLuckey, S. A.; Reid, G. E.; Wells, J. M.; Bundy, J. L. Ion/ion chemistry as a top-down approach for protein analysis. Curr. Opin. Biotech. 2002, 13, 57-64.

47. Hogan, J. M.; Pitteri, S. J.; McLuckey, S. A. Phosphorylation site identification via ion trap tandem mass spectrometry of whole protein and peptide ions: Bovine $\alpha$-crystallin A chain. Anal. Chem. 2003, 75, 6509-6516.

48. Wells, J. M.; Reid, G. E.; Engel, B. J.; Pan, P.; McLuckey, S. A. Dissociation reactions of gaseous ferro-, ferri-, and apo-cytochrome $c$ ions. J. Am. Soc. Mass Spectrom. 2001, 12, 873-876. 